\title{
Electron Spin Resonance of High-Spin Cobalt in Microporous Crystalline Cobalt-Containing Aluminophosphates
}

\author{
Bert M. Weckhuysen, ${ }^{* \dagger}$, An A. Verberckmoes, ${ }^{\dagger}$ Myriam G. Uytterhoeven, ${ }^{\dagger,}$ Frank E. Mabbs, ${ }^{\S}$ \\ David Collison, § Engelhardt de Boer," and Robert A. Schoonheydt ${ }^{\dagger}$ \\ Centrum voor Oppervlaktechemie en Katalyse, Departement Interfasechemie, K.U. Leuven, \\ Kardinaal Mercierlaan 92, 3001 Leuven, Belgium, EPSRC c.w. EPR Service Centre, Department of Chemistry, \\ University of Manchester, Oxford Road, MP13 9PL Manchester, United Kingdom, and Vakgroep Molecuul \\ Spectroscopie, Faculteit Natuurwetenschappen, Katholieke Universiteit Nijmegen, Toernooiveld, \\ 6525 ED Nijmegen, The Netherlands
}

Received: June 1, 1999; In Final Form: August 28, 1999

\begin{abstract}
Four highly crystalline, cobalt-containing microporous aluminophosphates (CoAPO-5, CoAPO-11, CoAPO44, and CoAPO-46) have been investigated by using liquid $\mathrm{He} \mathrm{X}$ - and/or Q-band electron spin resonance (ESR) spectroscopy in order to investigate the coordination of high-spin cobalt before and after calcination. The ESR spectra of the four zeolite structures are characterized by an axial signal with an effective $g_{\perp} \approx$ 5.80-5.44 and $g_{\|} \approx 2.00$. Quantitative temperature dependence measurements of this axial signal in the temperature range $4-30 \mathrm{~K}$ reveal a Curie-Weiss behavior for both as-synthesized and calcined samples confirming (a) the $m_{\mathrm{s}}= \pm 1 / 2$ ground state of magnetically isolated high-spin cobalt and (b) a zero field splitting $\Delta>0 \mathrm{~cm}^{-1}$. Quantitation of the ESR signals indicated that most of the $\mathrm{Co}^{2+}$ is ESR active and that only about $30 \%$ of this $\mathrm{Co}^{2+}$ can be oxidized to the ESR-inactive $\mathrm{Co}^{3+}$ after calcination. The spin Hamiltonian parameters of as-synthesized and calcined CoAPO-5 material, as determined by spectrum simulation and the microwave power saturation technique, support the presence of framework $\mathrm{Co}^{2+}$ in a flattened or elongated tetrahedron $\left(D_{2 d}\right)$.
\end{abstract}

\section{Introduction}

Microporous crystalline metalloaluminophosphates (MeAPO$n$ ) represent an important group of molecular sieves because of their potential catalytic and adsorptive properties., ${ }^{1,2}$ Many transition metal ions are thought to be incorporated in the framework of these molecular sieves. Only for $\mathrm{Co}^{2+}$ has unambiguous evidence been presented for isomorphous substitution. ${ }^{3,4}$

For example, the blue colored as-synthesized CoAPO-5 material is characterized by a typical triplet $\mathrm{d}-\mathrm{d}$ band system at around $17000 \mathrm{~cm}^{-1}$, which is assigned to tetrahedrally coordinated $\mathrm{Co}^{2+} .3,4$ Other evidence for tetrahedral high-spin $\mathrm{Co}^{2+}$ comes from X-ray absorption spectroscopy (XANES and EXAFS) and electron spin resonance (ESR) spectroscopy. ${ }^{6}$ Calcination in $\mathrm{O}_{2}$ creates a yellow-green sample and a completely new UV-Vis-NIR spectrum, as measured with diffuse reflectance spectroscopy (DRS). The triplet band system decreases in intensity and intense bands arise around 25200 and $31500 \mathrm{~cm}^{-1} \cdot{ }^{7-9}$ These bands are totally removed by a reduction treatment in, e.g., $\mathrm{H}_{2}, \mathrm{CO}$, and $\mathrm{NO}$ suggesting a partial oxidation of $\mathrm{Co}^{2+}$ to $\mathrm{Co}^{3+}$ after calcination in $\mathrm{O}_{2}$. Evidence for the partial oxidation of $\mathrm{Co}^{2+}$ to $\mathrm{Co}^{3+}$ was also found by ESR spectroscopy. ${ }^{8,9}$ Indeed, a $\mathrm{Co}^{2+}$ ESR signal of reduced intensity in calcined CoAPO-5 material suggests an oxidation to the ESR

* To whom correspondence should be addressed. E-mail: Bert.Weckhuysen@agr.kuleuven.ac.be.

$\dagger$ K.U. Leuven.

$\doteqdot$ Present address: S.I.S.-consult, Rotpoelstraat 361, B-3001 Leuven, Belgium.

§ University of Manchester.

"Katholieke Universiteit Nijmegen. inactive $\mathrm{Co}^{3+}$. Lee and Chon proved the oxidative abilities of calcined CoAPO-5 and CoAPO-11 with ESR by the formation of radicals from alkenes. ${ }^{10}$

However, this oxidation-reduction behavior of CoAPO-5 materials has been challenged by Kurshev et al. ${ }^{11}$ They observed an unusual temperature-dependent behavior for the $\mathrm{Co}^{2+} \mathrm{ESR}$ signal of calcined CoAPO-5 (non Curie-Weiss behavior) in comparison with the as-synthesized CoAPO-5 material (CurieWeiss behavior) between 4 and $20 \mathrm{~K}$, and accordingly, a zerofield splitting $\Delta$ of $-13 \mathrm{~cm}^{-1}$ was determined for the calcined material. This observation was explained by a distortion of tetrahedral $\mathrm{Co}^{2+}$ to dihedral symmetry resulting from the interaction with two $\mathrm{O}_{2}$ molecules. Also, Pereira et al. ${ }^{12}$ and Berndt et al. ${ }^{13}$ suggested a distortion of framework $\mathrm{Co}^{2+}$ to be the cause of the color change from blue to yellow-green instead of a (partial) oxidation to $\mathrm{Co}^{3+}$.

In view of this controversy about the oxidation ability of $\mathrm{Co}^{2+}$ to $\mathrm{Co}^{3+}$ in the lattice of CoAPOs, we undertook a detailed ESR study of a series of highly crystalline CoAPO- $n$ samples (CoAPO-5, CoAPO-11, CoAPO-44, and CoAPO-46). Temperature-dependent and quantitative ESR measurements were performed for both the as-synthesized and calcined CoAPOs. In addition, a continuous wave (cw) microwave saturation technique was used for determining the zero-field splitting $\Delta$. This method has been first described by Makinen and coworkers and further on applied on $\mathrm{Co}^{2+} \cdot{ }^{14-16}$ Finally, the spin Hamiltonian parameters were estimated by simulation of the ESR spectra. 
TABLE 1: Chemical Composition of the Samples Used for ESR Measurements in This Work

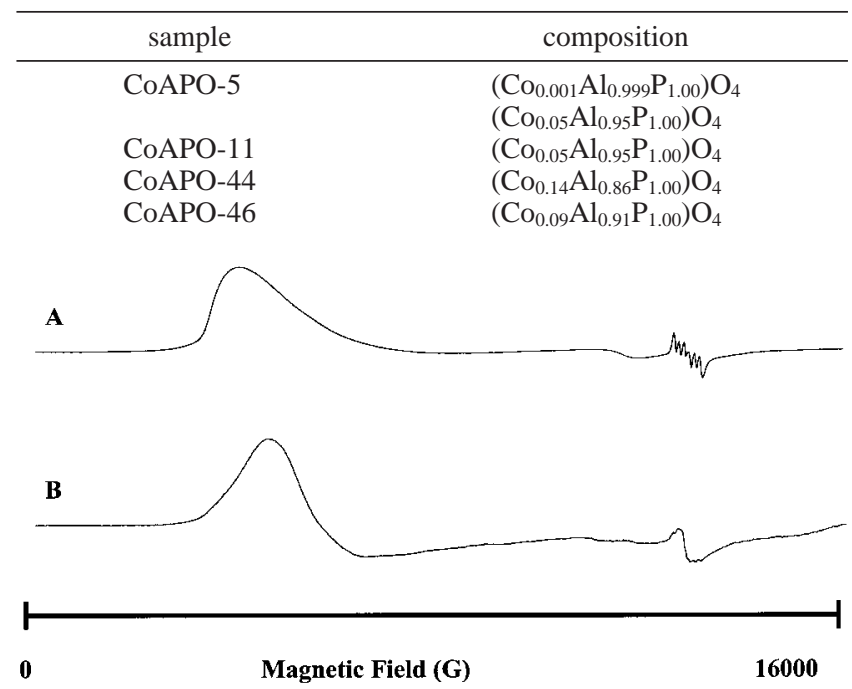

Figure 1. Experimental Q-band ESR spectrum of (A) as-synthesized and (B) calcined $\mathrm{Co}_{0.05} \mathrm{Al}_{0.95} \mathrm{P}_{1} \mathrm{O}_{4}-5(T=10 \mathrm{~K}, P=2.34 \mathrm{~mW})$.

\section{Experimental Section}

CoAPO-5, CoAPO-11, CoAPO-44, and CoAPO-46 samples were synthesized as described in previous publications, ${ }^{3,17}$ and their chemical composition is summarized in Table 1. In all cases phase-pure CoAPO- $n$ materials with excellent crystallinity were obtained. Calcination was performed in $\mathrm{O}_{2}$ at $500{ }^{\circ} \mathrm{C}$ in an ESR flow cell. The samples were cooled in $\mathrm{O}_{2}$ and flushed with He before measuring. ESR measurements were performed directly after pretreatment. The ESR spectra were recorded either at X- $(\approx 9.5 \mathrm{GHz})$ or Q-band $(\approx 35 \mathrm{GHz})$ on a Bruker ESP300E spectrometer with an Oxford Instruments liquid Helium cryostat in the temperature range $4-300 \mathrm{~K}$. The calcined $\mathrm{Co}_{0.001-}$ $\mathrm{Al}_{0.999} \mathrm{P}_{1.00} \mathrm{O}_{4}$ sample was used for power saturation studies at 7.2, 9.7, and $12 \mathrm{~K}$ in the microwave power range $0.1-533 \mathrm{~mW}$ at X-band. ESR simulations were performed by simulation programs developed by Mabbs and Collison. ${ }^{18,19}$ ESR intensities of the as-synthesized and calcined samples were obtained by double integration of the ESR spectra obtained under nonsaturating conditions. The whole magnetic field sweep range was integrated for X-band spectra, whereas only the " $g_{\perp}$ " was used for integration of the Q-band spectra.

\section{Results}

1. ESR Signals of $\mathrm{Co}^{2+}$ in As-Synthesized and Calcined CoAPO-n. Both X- and Q-band ESR spectra of the assynthesized and calcined CoAPO- $n$ materials were measured between 4 and $300 \mathrm{~K}$. As an example, we will discuss the Q-band spectra of $\mathrm{Co}_{0.05} \mathrm{Al}_{0.95} \mathrm{P}_{1} \mathrm{O}_{4}$ in more detail. Figure 1 shows the ESR spectra of the as-synthesized and calcined $\mathrm{Co}_{0.05}$ $\mathrm{Al}_{0.95} \mathrm{P}_{1} \mathrm{O}_{4}-5$ measured in Q-band at $10 \mathrm{~K}$. Similar ESR spectra were obtained for other measurement temperatures, however, with different overall signal intensities. The ESR spectrum of the as-synthesized sample (Figure 1A) is characterized by a broad, axially symmetric signal with an effective $g_{\perp} \approx 5.80-$ 5.44 and $g_{\|} \approx 2.00$. In addition, a small but characteristic signal around $g_{\text {eff }}=2$ due to $\mathrm{Mn}^{2+19}$ is present in this sample, which was almost invisible at X-band frequency. Similar ESR spectra were obtained for the CoAPO-11, CoAPO-44, and CoAPO-46 materials, as shown in Figure 2, although the CoAPO-46 sample has an extraderivative line in the ESR spectrum at around $g=$ 2.1. In any case, the observed effective $g$ values are comparable
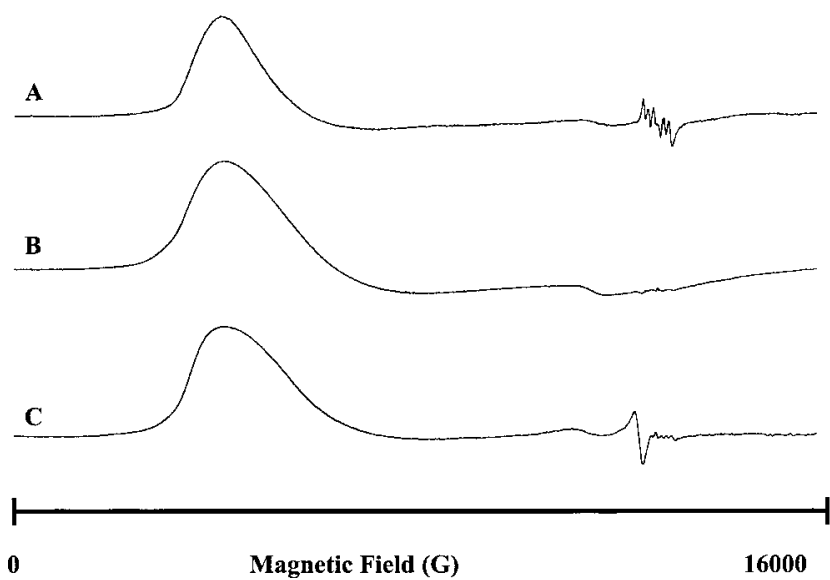

Figure 2. Experimental Q-band ESR spectra of as-synthesized (A) CoAPO-11, (B) CoAPO-44, and (C) CoAPO-46 ( $T=10 \mathrm{~K}, P=2.34$ $\mathrm{mW})$.

to those previously found for CoAPO-5 and CoAPO-41 and comparable with those of relevant reference compounds (Table 2). ${ }^{8,11,20-24}$ After calcination, the overall ESR signal intensity decreases and the perpendicular component of the broad, axially symmetric signal shifts to 5.44 (Figure 1B).

To evaluate the oxidation ability of $\mathrm{Co}^{2+}$ to the ESR-inactive $\mathrm{Co}^{3+}$ in the lattice of CoAPOs, we have plotted the ESR intensities of the as-synthesized and calcined materials in Figure 3 as a function of the Co content (Table 1). Except for the samples with the lowest Co content, the ESR intensities increase linearly with the Co loading. The straight line extrapolates to zero within the experimental accuracy limits. This is true for the as-synthesized and the calcined samples. After calcination, however, the overall ESR signal intensities decrease by about $30 \%$ for all the samples. It is important to stress that a reliable quantification of the ESR spectra is difficult because the $\mathrm{Co}^{2+}$ signals are smeared out over a large magnetic field range. The latter was much more pronounced for the Q-band ESR spectra. The observed decrease of signal intensity with $30 \%$ is significant, knowing that the absolute and relative error for ESR quantifications are estimated to be about $20 \%$ and $5-10 \%$, respectively. ${ }^{25,26}$ These results confirm those of Kurshev et al.,"1 Pereira et al. ${ }^{12}$ and Berndt et al. ${ }^{13}$ that only a small fraction of $\mathrm{Co}^{2+}$ is oxidized to $\mathrm{Co}^{3+}$ during calcination.

Simulations of the ESR spectra result in true $g, D$, and $E$ values for a tetrahedral $\mathrm{Co}^{2+}$ ion in distorted symmetry $\left(D_{2 d}\right) .{ }^{19}$ An example of a simulated spectrum is shown in Figure 4 for as-synthesized CoAPO-5 with $0.05 \mathrm{Co}$. The spin Hamiltonian parameters obtained are given in Table 3. At this stage in the analysis, we can state that (a) a minimum, absolute zero field splitting $(2|D|)$ of around $7 \mathrm{~cm}^{-1}$ is necessary in order to simulate the experimental ESR spectra, (b) the sign of $D$ is not determined, (c) axial ESR symmetry has been assumed, and (d) the exact line shape of the experimental ESR spectrum could not be reproduced, even by assuming a rhombic ESR symmetry in the simulation. The latter suggests that several $\mathrm{Co}^{2+}$ sites, slightly differing in coordination geometry, are contributing to the overall ESR powder spectrum.

2. Temperature Dependence of the ESR Signals of $\mathrm{Co}^{2+}$. To understand the significance of temperature-dependent ESR measurements, it is necessary to explain the ligand field energy level diagram of the ground state ${ }^{4} \mathrm{~A}_{2}$ of high-spin $\mathrm{Co}^{2+}$ (Figure 5). Distortion of the tetrahedron will split the ${ }^{4} \mathrm{~A}_{2}$ ground state into two Kramers doublets $\left(m_{\mathrm{s}}= \pm 1 / 2\right.$ and $\left.m_{\mathrm{s}}= \pm 3 / 2\right)$, which are degenerate in the absence of a magnetic field $\mathrm{H}$. This splitting is called the zero field splitting (ZFS) $\Delta$. The 
TABLE 2: Effective ESR Parameters of $\mathrm{Co}^{2+}$ in $\mathrm{CoAP}(\mathrm{S}) \mathrm{O}-n$ Molecular Sieves and in Some Other Oxide Lattices

\begin{tabular}{|c|c|c|c|c|}
\hline sample & pretreatment & temp $(\mathrm{K})$ & effective $g$ values & reference \\
\hline \multirow[t]{4}{*}{ CoAPO-5 } & as-synthesized & 7 & $g_{\perp}=6.2 ; g_{\|}=2.26$ & 11 \\
\hline & calcined & 7 & $g_{\perp}=5.45 ; g_{\|}=\mathrm{nd}^{a}$ & \\
\hline & as-synthesized & 10 & $g_{\perp}=5.80 ; g_{\|}=2.00$ & this work \\
\hline & calcined & 10 & $g_{\perp}=5.44 ; g_{\|}=2.00$ & \\
\hline \multirow{2}{*}{ CoAPSO-34 } & as-synthesized & 4.2 & $g_{\perp}=6.2 ; g_{\|}=2.25$ & 8 \\
\hline & calcined & 4.2 & $g_{\perp}=5.52 ; g_{\|}=2.25$ & \\
\hline \multirow[t]{2}{*}{ CoAPSO-41 } & as-synthesized & 4.5 & $g_{\perp}=6.075 ; g_{\|}=2.057$ & 20 \\
\hline & calcined & 4.5 & $g_{\perp}=5.560 ; g_{\|}=2.061$ & \\
\hline $\mathrm{Co}^{2+}$ in $\mathrm{TiO}_{2}$ & $\mathrm{na}^{b}$ & 4.2 & $g_{z z}=5.860 ; g_{x x}=2.090 ; g_{y y}=3.725$ & 21 \\
\hline $\mathrm{Co}^{2+}$ in $\mathrm{KTaO}_{3}$ & $\mathrm{na}^{b}$ & 4.2 & $g_{\perp}=4.958 ; g_{\|}=2.067$ & 22 \\
\hline $\mathrm{Co}^{2+}$ in $\mathrm{MgTiO}_{3}$ & $\mathrm{na}^{b}$ & 4.2 & $g_{\|}=2.861 ; g_{\perp}=4.990$ & 23 \\
\hline \multirow[t]{2}{*}{$\mathrm{Co}^{2+}$ in $\alpha-\mathrm{Al}_{2} \mathrm{O}_{3}$} & $\mathrm{na}^{b}$ & 4.2 & $g_{\perp 1}=4.947 ; g_{\| 1}=2.292$ & 24 \\
\hline & & & $g_{\perp 2}=4.855 ; g_{\| 2}=2.808$ & \\
\hline
\end{tabular}

${ }^{a}$ nd $=$ not determined. ${ }^{b}$ na $=$ not applicable.

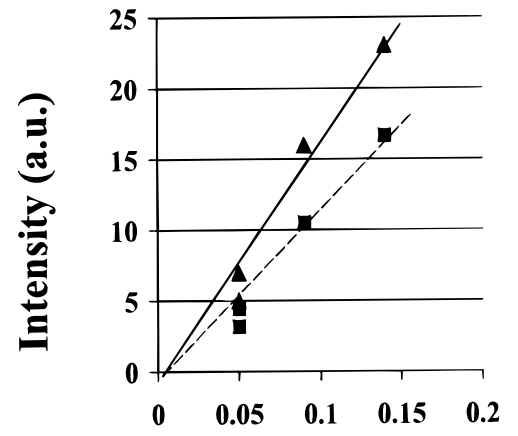

Co-content (x)

Figure 3. Intensity of ESR spectra of as-synthesized $(\mathbf{\Lambda})$ and calcined (ロ) $\mathrm{Co}_{x} \mathrm{Al}_{1-x} \mathrm{P}_{1} \mathrm{O}_{4}-n$ materials (with $n=5,11,44$, and 46): Doubly integrated ESR spectrum intensity of $\mathrm{Co}^{2+}$ as a function of the Co content $x(T=10 \mathrm{~K}, P=2.34 \mathrm{~mW})$.
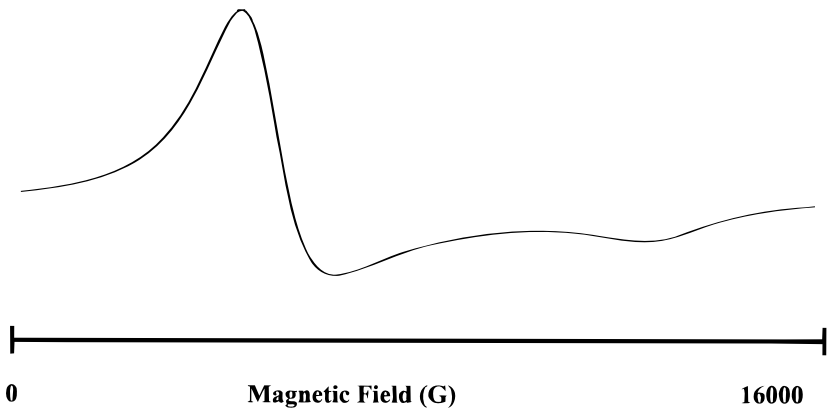

Figure 4. Simulated Q-band ESR spectrum of as-synthesized CoAPO5. (The spin Hamiltonian parameters are given in Table 3.)

TABLE 3: Simulated $g, D$, and $E$ Values of As-Synthesized and Calcined CoAPO-5 ${ }^{18,19}$

\begin{tabular}{llc}
\hline \multicolumn{1}{c}{ treatment } & \multicolumn{1}{c}{$g$ values } & $\begin{array}{c}D \text { and } E \text { values } \\
\left(\mathrm{cm}^{-1}\right)\end{array}$ \\
\hline as-synthesized & $g_{x x}=2.643 ; g_{y y}=2.643 ; g_{z z}=2.020$ & $D=3.5 ; E=0.0$ \\
calcined & $g_{x x}=2.443, g_{y y}=2.443 ; g_{z z}=2.020$ & $D=3.5 ; E=0.0$
\end{tabular}

corresponding energy splitting between the two doublets is given by $2\left(D^{2}+3 E^{2}\right)^{1 / 2}$, with $D$ and $E$ the zero field splitting parameters. The sign of $\Delta$ is determined by whether the $m_{\mathrm{s}}=$ $\pm 1 / 2$ and $m_{\mathrm{s}}= \pm 3 / 2$ doublet lies lowest. The value of $\Delta$ is positive when $m_{\mathrm{s}}= \pm 1 / 2$ is the lowest and negative when $m_{\mathrm{s}}$ $= \pm 3 / 2$ is the lowest. In the presence of a magnetic field $\mathrm{H}$, the two degenerate doublets are split, and incident microwave energy will induce transitions according to the selection rule $\Delta m_{\mathrm{s}}= \pm 1$. The different possible transitions, giving rise to an ESR signal, are given in Figure 5. Depending on the sign of $\Delta$,
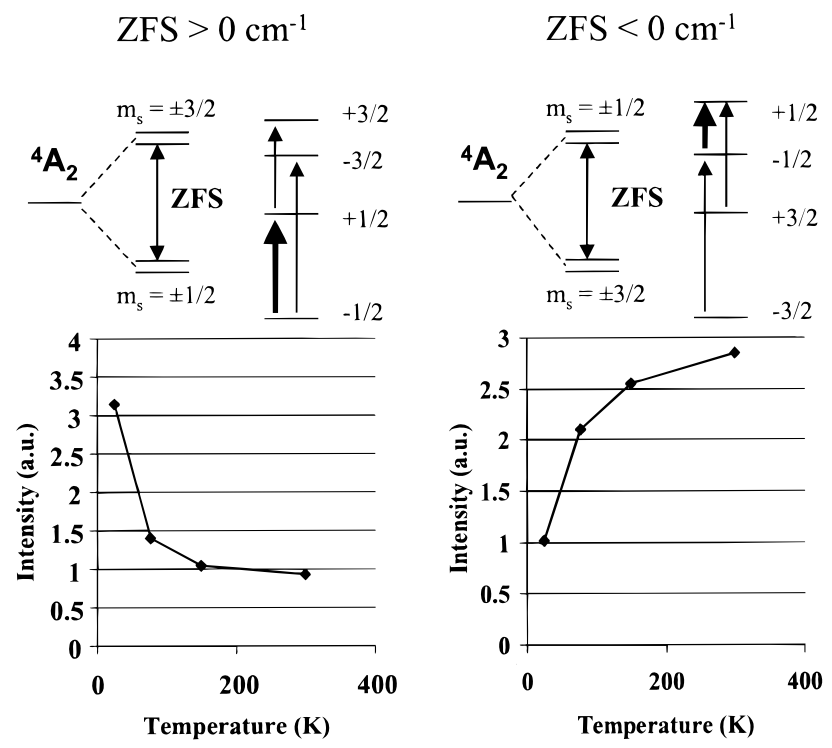

Figure 5. Ligand field energy diagram for high-spin tetrahedral $\mathrm{Co}^{2+}$ for two different cases, i.e., the zero field splitting (ZFS) larger or smaller than $0 \mathrm{~cm}^{-1}$. The intensity vs temperature plots were obtained by generating simulated powder spectra with an isotropic $g$ value equal to 2.00 as a function of the temperature; isotropic line widths $=50$ $\mathrm{mT}$ and $v=35.000 \mathrm{GHz}$.

a different temperature behavior will be observed. Positive values give rise to Curie-Weiss behavior, whereas negative values result in non-Curie-Weiss behavior. The latter was experimentally observed by Kurshev et al. ${ }^{11}$

Figure 6 shows the intensity of the $\mathrm{Co}^{2+}$ signals in assynthesized and calcined $\mathrm{Co}_{0.05} \mathrm{Al}_{0.95} \mathrm{P}_{1} \mathrm{O}_{4}$ as a function of the temperature measured in the range $4-30 \mathrm{~K}$ for nonsaturating conditions. This has been done by double integration of the Q-band ESR spectra measured at the different temperatures over the magnetic field range 0-16000 G (Figure 6A). Figure 6B shows that a clear Curie-Weiss behavior was obtained for both the as-synthesized and calcined $\mathrm{Co}_{0.05} \mathrm{Al}_{0.95} \mathrm{P}_{1} \mathrm{O}_{4}$. This suggests that the value of $\Delta$ is positive and that the $m_{\mathrm{s}}= \pm 1 / 2$ doublet is lowest in energy. Similar results were obtained for CoAPO$11,-44$, and -46 .

3. Microwave Saturation Technique. As was pointed out by Banci et al., ${ }^{27}$ effective $g$ factors, as presented in Table 2, do not distinguish between distorded octahedral, pentacoordinate, and tetrahedral cobalt sites. This can only be done by determining the value of the zero-field parameter $\Delta=2\left(D^{2}+\right.$ $\left.3 E^{2}\right)^{1 / 2}$, which is considered as a diagnostic signature of the coordination geometry of $\mathrm{Co}^{2+} \cdot{ }^{26}$ To determine the zero-field parameter $\Delta$ of a calcined CoAPO-5 sample, we have used the 

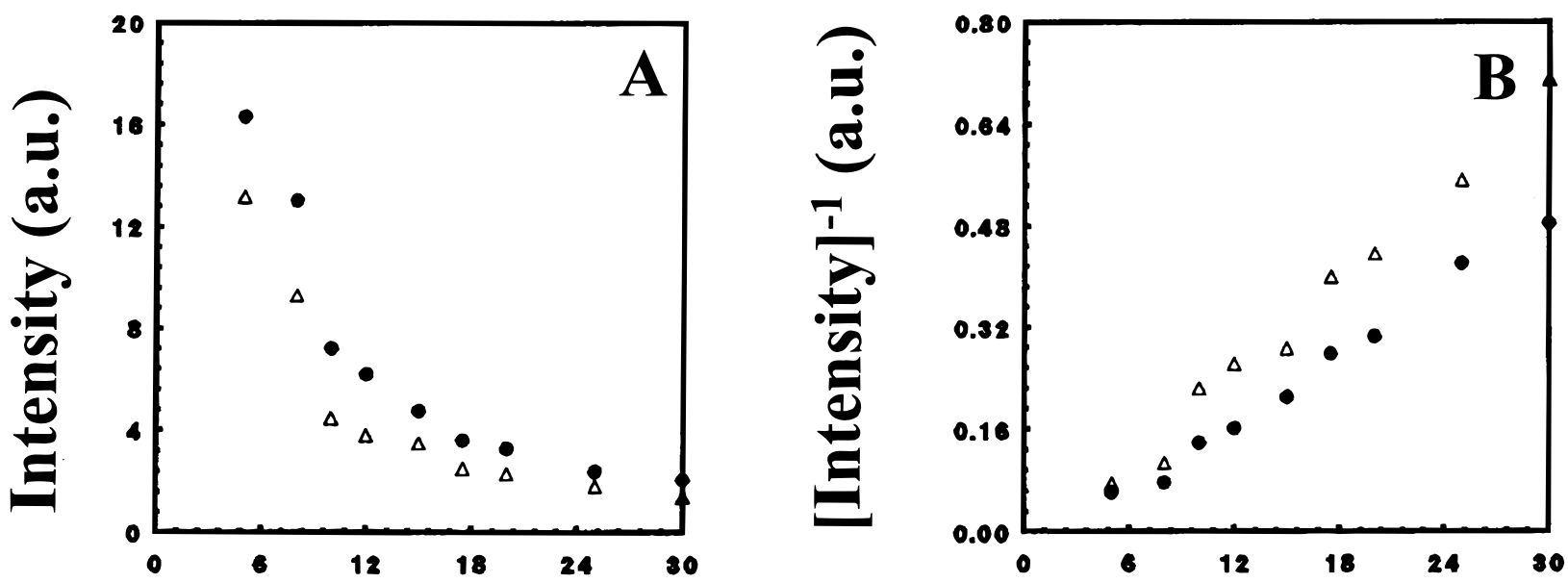

\section{Temperature (K)}

Figure 6. Doubly integrated ESR spectrum intensity (A) and reciprocal intensity (B) of $\mathrm{Co}^{2+}$ ions in as-synthesized $(\bullet)$ and calcined $(\triangle) \mathrm{Co}_{0.05}{ }^{-}$ $\mathrm{Al}_{0.95} \mathrm{P}_{1} \mathrm{O}_{4}$ from 4 to $30 \mathrm{~K}(P=2.34 \mathrm{~mW})$.

constant microwave saturation technique. Such study requires a CoAPO-5 material with a very low Co content, i.e., $\mathrm{Co}_{0.001^{-}}$ $\mathrm{Al}_{0.999} \mathrm{P}_{1} \mathrm{O}_{4}$. The principle of the microwave saturation technique was first described by Yim et al. for Fe-containing metalloproteins ${ }^{14}$ and later extensively applied by Makinen et al. for $\mathrm{Co}^{2+} .{ }^{15,16}$ Makinen et al. observed that the splitting $(\Delta)$ between the two lowest Kramers doublets of high-spin $\mathrm{Co}^{2+}$ (Figure 5) follows the order $\Delta_{4}<\Delta_{5}<\Delta_{6}$, where the subscripts designate the coordination number. ${ }^{15,16}$ The range of $\Delta$ values was found to be $<13 \mathrm{~cm}^{-1}$ in tetracoordinate sites, $20-50 \mathrm{~cm}^{-1}$ in pentacoordinate sites, and $>50 \mathrm{~cm}^{-1}$ in hexacoordinate sites. These values will be taken as diagnostic reference values for the $\Delta$ values determined in this paper.

The principle of the microwave saturation technique is based on the relationship between $P_{1 / 2}$ and the spin-lattice $\left(T_{1}\right)$ and spin-spin relaxation time $\left(T_{2}\right)$ of the paramagnetic system given in eq $1 .^{14-16}$

$$
P_{1 / 2} \propto\left(1 / T_{1} T_{2}\right)
$$

with $P_{1 / 2}$ the saturating microwave power that results in half of the normal ESR signal intensity. If $1 / T_{2}$ is constant within the temperature range under study, which is achieved by high dilution of the paramagnetic centers, then $P_{1 / 2}$ is only proportional to $1 / T_{1}$. Moreover, if the spin-lattice relaxation process corresponds to the Orbach process, ${ }^{28,29}$ one can write that

$$
1 / T_{1} \propto \exp (-\Delta / k T)
$$

which results in

$$
P_{1 / 2}=A \exp (-\Delta / k T) \quad \text { or } \quad \ln P_{1 / 2}=\ln A-(\Delta / k T)
$$

with $A$ a proportionality constant characteristic of the phononspin properties, $k$ the Boltzmann constant, and $\Delta$ the zero-field splitting. Thus, by plotting $\ln P_{1 / 2}$ as a function of $1 / T$, one can determine the zero field splitting $\Delta$.

This theory has been applied in Figures 7 and 8 for calcined $\mathrm{Co}_{0.001} \mathrm{Al}_{0.999} \mathrm{P}_{1} \mathrm{O}_{4}$ measured at 7.2, 9.7, and $12 \mathrm{~K}$. Figure 7 was obtained by plotting the signal amplitude $(S)$ of the ESR spectrum at $g_{\text {eff }}=5.44$, as $\log \left(S P^{-1 / 2}\right)$, versus $\log P$, with $P$

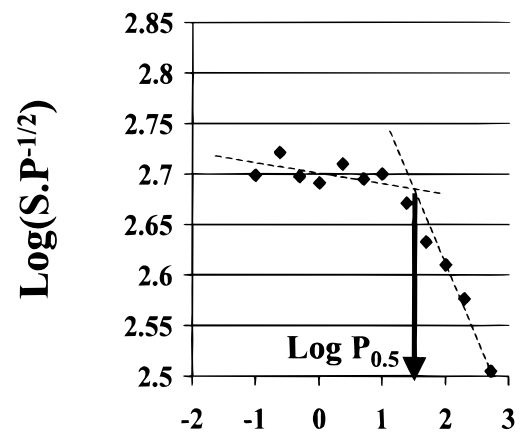

\section{$\log P$}

Figure 7. $\log \left(S P^{-1 / 2}\right)$ versus $\log P$ at $12 \mathrm{~K}$ for calcined $\mathrm{Co}_{0.001^{-}}$ $\mathrm{Al}_{0.999} \mathrm{P}_{1} \mathrm{O}_{4}$ (with $S=$ signal amplitude of the ESR spectrum at $g_{\text {eff }}=$ 5.44 and $P=$ the microwave power).

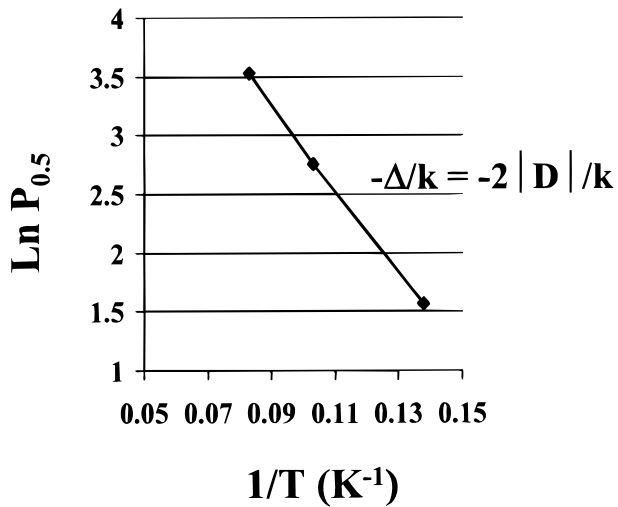

Figure 8. Semilogaritmic plot of $\ln P_{0.5}$ versus $1 / T$ for calcined $\mathrm{Co}_{0.001^{-}}$ $\mathrm{Al}_{0.999} \mathrm{P}_{1} \mathrm{O}_{4}$.

the microwave power at $12 \mathrm{~K}$. Figure 7 can be interpreted in terms of two straight lines: (1) at low microwave power $\log \left(S P^{-1 / 2}\right)$ is almost constant because there is no signal saturation; (2) a tangent to the curves can be drawn at high microwave power where there is saturation of the ESR signal. The point of intersection between the two lines gives $\log P_{0.5}$. The semilogarithmic plot of $\ln P_{0.5}$ versus $1 / T$ results in a straight 
line with slope $-\Delta / k$ (Figure 8 ). One obtains then that $2|D|$ is equal to $7 \mathrm{~cm}^{-1}$, which is equal to the value which has been used for simulating the experimental ESR spectra (Table 3 and Figure 4).

Finally, the validity of the applied method has to be verified. Indeed, the use of the microwave saturation technique is subject to the following conditions.

(1) $\Delta>k T \gg h v$.

(2) The magnetic susceptibility is a function of the spinlattice relaxation $T_{1}$ only.

The first condition can be checked as follows: (a) $h v=6.08$ $\times 10^{-24} \mathrm{~J}$; (b) $k T(7.2 \mathrm{~K})=9.93 \times 10^{-23} \mathrm{~J}, k T(9.7 \mathrm{~K})=1.34$ $\times 10^{-23} \mathrm{~J}$, and $k T(12 \mathrm{~K})=1.66 \times 10^{-22} \mathrm{~J}$; (c) $\Delta=7 \mathrm{~cm}^{-1}=$ $1.39 \times 10^{-22} \mathrm{~J}$. One can see that condition (1) is fulfillled at $7.2 \mathrm{~K}$ and is at the limit for $9.7 \mathrm{~K}$. In that case the plot of $\ln$ $P_{0.5}$ versus $1 / T$ is not linear, and application of the Orbach approximation results in an overestimate of the zero field splitting energy, a problem which was previously described by Yim et al. ${ }^{14}$

The second condition can be verified experimentally. The following conditions have to be fulfilled: (1) the slope of the line is invariant with temperature; (2) no increase in line width with temperature; (3) no change in line width by a saturating microwave power. All these conditions were fulfilled in our experiments, and thus the value obtained for $\Delta$ is valid, within the experimental accuracy of the experiments.

\section{Discussion}

All the CoAPO- $n$ (where $n=5,11,44$, or 46) samples reported in this study are highly crystalline and without additional phases, which might complicate the interpretation of the spectroscopic data. They have, with the exception of $\mathrm{Co}_{0.001^{-}}$ $\mathrm{Al}_{0.999} \mathrm{P}_{1.00} \mathrm{O}_{4}-5$, been studied in detail by $\mathrm{X}$-ray diffraction, scanning electron microscopy and diffuse reflectance spectroscopy in the past. ${ }^{3,17}$ For example, the blue-colored as-synthesized materials all show a typical triplet $\mathrm{d}-\mathrm{d}$ band system at around $17000 \mathrm{~cm}^{-1}$, which is assigned to tetrahedrally coordinated $\mathrm{Co}^{2+}$ substituting for $\mathrm{Al}^{3+}$ in the lattice. ${ }^{3,4}$ Calcination in $\mathrm{O}_{2}$ creates a yellow-green-colored sample with a triplet band reduced in intensity in DRS, and two new intense absorption bands at around 25200 and $31500 \mathrm{~cm}^{-1}$. The present ESR investigation allows the discussion of two unresolved issues in the literature: (1) the oxidation state of $\mathrm{Co}^{\mathrm{n}+}(n=2$ and/or 3$)$ in calcined CoAPO- $n$ molecular sieves; (2) the coordination environment of $\mathrm{Co}^{2+}$ in as-synthesized and calcined CoAPO- $n$ molecular sieves. It is important to notice that during the course of this work an interesting paper on CoAPO-5 materials appeared in the literature. ${ }^{30}$ This recent paper by Howe and coworkers is addressing similar questions on the coordination chemistry of $\mathrm{Co}^{2+}$ in CoAPO- $n$ materials by using a combination of UV-vis DRS, X-ray absorption spectroscopy (EXAFSXANES), and ESR spectroscopy. We will compare their results with those presented in this paper.

As was already pointed out in the literature, the evidence for oxidation of $\mathrm{Co}^{2+}$ to $\mathrm{Co}^{3+}$ during calcination includes (1) changes in the DRS spectra; (2) a shortening of the $\mathrm{Co}-\mathrm{O}$ bond length, as determined by EXAFS; (3) a reduction in intensity of the $\mathrm{Co}^{2+}$ ESR signal, and (4) the presence in calcined CoAPO- $n$ samples of oxidizing sites capable of forming cation radicals.

However, all these arguments have to be taken with care. Indeed, the present investigation indicates that the reduction in intensity of the $\mathrm{Co}^{2+}$ ESR signal during calcination is small (about 30\%), but systematic, for all the samples. It can be considered as a significant effect, even when the absolute accuracy of the signal intensities is only $20 \%$. Our observation is in line with those of Kurshev et al., ${ }^{11}$ Pereira et al., ${ }^{12}$ and Berndt et al. ${ }^{13}$ that only a small fraction of $\mathrm{Co}^{2+}$ is oxidized during calcination. This observation is not in full agreement with the results of Thomson et al. ${ }^{30}$

The observed change in ESR parameters and line shape of the $\mathrm{Co}^{2+}$ ESR signal clearly indicates a change in the coordination geometry upon calcination, but it is not necessary to postulate the extreme distortion causing negative zero-field splitting $\left(2|D|=-13 \mathrm{~cm}^{-1}\right)$ and a non-Curie-Weiss behavior as proposed by Kurshev et al. ${ }^{11}$ Thus, in contrast to Kurshev et al., ${ }^{11}$ we found a zero field splitting $\Delta=2|D|=7 \mathrm{~cm}^{-1}$, and a normal ESR temperature dependency for both the assynthesized and calcined CoAPO- $n$ material. The latter has also been observed by Thomson et al. ${ }^{30}$ This confirms that the results of Kurshev et al. ${ }^{11}$ cannot be reproduced.

Using the criterion of Makinen et al., ${ }^{15,16}$ the present work indicates four-coordinate $\mathrm{Co}^{2+}$. We believe that this fourcoordination seems most realistic because (1) it is the original configuration of as-synthesized CoAPO- $n$ material and (2) normally $\mathrm{O}_{2}$ molecules are not a strong ligand and therefore does not influence the coordination number. The model, which best explains the observations is that proposed by Barret et al., ${ }^{6}$ namely that charge compensation in the calcined CoAPO- $n$ materials is achieved through the formation of anion vacancies following loss of protonated template. Such anion vacancy formation will distort the framework in the proximity of $\mathrm{Co}^{2+}$, causing the change in ESR parameters (Table 3) and the observed effect on the line shape of the ESR signal. The distorted symmetry can be explained by the presence of an elongated or flattened tetrahedron of $\mathrm{Co}^{2+}\left(D_{2 d}\right)$.

A remaining issue concerns the changes in the DRS spectra upon calcination. Thus, future research has to be directed toward a more accurate interpretation of the observed absorption bands around 25200 and $31500 \mathrm{~cm}^{-1}$ in the DRS spectrum of calcined CoAPO-5. Hopefully, quantum chemical calculations, based on DFT-CASPT2 methods, may resolve this issue soon. ${ }^{31}$

\section{Conclusions}

The main conclusions from this work are as follows.

Quantitative temperature-dependent ESR measurements of $\mathrm{Co}^{2+}$ in CoAPO- $n$ reveal a Curie-Weiss behavior for both assynthesized and calcined materials confirming (a) the $m_{\mathrm{s}}= \pm 1 / 2$ ground state of magnetically isolated high-spin cobalt and (b) a zero field splitting $\Delta>0 \mathrm{~cm}^{-1}$.

Quantitation of the ESR spectra indicated that most of the $\mathrm{Co}^{2+}$ is ESR visible and that only a small part of the $\mathrm{Co}^{2+}$ is oxidized to $\mathrm{Co}^{3+}$ after calcination.

Simulations of the ESR spectra and application of the microwave saturation technique point towards an axial symmetry for $\mathrm{Co}^{2+}$ with a zero field splitting $\Delta$ of $7 \mathrm{~cm}^{-1}$. This observation can be explained by the presence of $\mathrm{Co}^{2+}\left(D_{2 d}\right)$ as either an elongated or flattened tetrahedron. Such a site is most probably located near an oxygen vacancy in the zeolite lattice.

Acknowledgment. B.M.W. acknowledges a postdoctoral fellowship of the FWO-Vlaanderen. A.A.V. and M.G.U. acknowledge a research grant of the IWT. This work was financially supported by the Geconcerteerde Onderzoeksactie (GOA) of the Flemish Government, by the Fonds voor Kollectief Fundamenteel Onderzoek (FKFO) and by the FWO-British Council Grant 7.003.97N. 


\section{References and Notes}

(1) Weckhuysen, B. M.; Rao, R. R.; Martens, J. A.; Schoonheydt, R. A. Eur. J. Inorg. Chem. 1999, 565.

(2) Hartmann, M.; Kevan, L. Chem. Rev. 1999, 99, 635.

(3) Verberckmoes, A. A.; Uytterhoeven, Schoonheydt, R. A. Zeolites 1997, 19, 180

(4) Verberckmoes, A. A.; Weckhuysen, B. M.; Schoonheydt, R. A Microporous Mesoporous Mater. 1998, 22, 165.

(5) Peeters, M. P. J.; van Hoof, J. H. C.; Sheldon, R. A.; Zholobenko,

V. L.; Kustov, L. M.; Kazansky, V. B. In Proceedings of the 9th International Zeolite Conference, Montreal; Higgins, J. B., Van Balmoos, R., Treacy, M. M. J., Eds.; Butterworth-Heinemann: New York, 1992; p 627.

(6) Barret, P. A.; Sankar, G.; Catlow, C. R. A.; Thomas, J. M. J. Phys. Chem. 1996, 100, 8977.

(7) Schoonheydt, R. A.; De Vos, R.; Pelgrims, J.; Leeman, H. In Zeolites: Facts, Figures, Future; Jacobs, P. A., Vansanten, R. A., Eds.; Elsevier: Amsterdam, 1989; p 559.

(8) Iton, L. E.; Choi, I.; Desjardins, J. A.; Maroni, V. A. Zeolites 1989 9, 535 .

(9) Montes, C.; Davis, M. E.; Murray, B.; Narayna, M. J. Phys. Chem. 1990, 94, 6425 .

(10) Lee, Y. J.; Chon, H. J. Chem. Soc., Faraday Trans. 1996, 92, 3453.

(11) Kurshev, V.; Kevan, L.; Parillo, D. J.; Pereira, C.; Kokotailo, G. T.; Gorte, R. J. J. Phys. Chem. 1994, 98, 10160.

(12) Pereira, C.; Parillo, D. J.; Kokotailo, G. T.; Gorte, R. J. In Proceedings of the 9th International Zeolite Conference, Montreal; Higgins, J. B., Van Balmoos, R., Treacy, M. M. J., Eds.; Butterworth-Heinemann: New York, 1992; extended abstracts, p 125.

(13) Berndt, H.; Martin, A.; Zhang, Y. Microporous Mater. 1996, 6, 1.

(14) Yim, M. B.; Kuo, L. C.; Makinen, M. W. J. Magn. Reson. 1982, 46, 247.
(15) Makinen, M. W.; Kuo, L. C.; Yim, M. B.; Wells, G. B.; Fukujama, J. M.; Kim, J. E. J. Am. Chem. Soc. 1985, 107, 5245.

(16) Makinen, M. W.; Kuo, L. C.; Yim, M. B.; Wells, G. B.; Fukujama,

J. M.; Kim, J. E. J. Am. Chem. Soc. 1985, 107, 5255.

(17) Gao, X.; Weckhuysen, B. M.; Schoonheydt, R. A. Microporous Mesoporous Mater. 1999, 27, 75.

(18) Collison, D.; Mabbs, F. E. J. Chem. Soc., Dalton Trans. 1982, 1565.

(19) Mabbs, F. E.; Collison, D. Electron paramagnetic resonance of d transition metal compounds; Elsevier Science: Amsterdam, 1992.

(20) Prakash, A. M.; Hartmann, M.; Kevan, L. J. Phys. Chem. B 1997, $101,6819$.

(21) Zverev, G. M.; Prokhorov, A. M. Sov. Phys. JETP 1963, 16, 303.

(22) Abragam, M. M.; Boatner, L. A.; Olson, D. N.; Hochli, U. T. J. Chem. Phys. 1984, 81, 2528.

(23) Haider, A. F. M. Y.; Edgar, A. J. Phys. C 1980, 13, 6239.

(24) Zverev, G. M.; Prokhorov, A. M. Sov. Phys. JETP 1959, 9, 451. Zverev, G. M.; Prokhorov, A. M. Sov. Phys. JETP 1961, 12, 41.

(25) Weckhuysen, B. M.; Heidler, R.; Schoonheydt, R. A. In Molecular Sieves-Science and Technology; Springer-Verlag: Berlin, in press.

(26) Pilbrow, J. R. Transition Ion Electron Paramagnetic Resonance; Clarendon Press: Oxford, 1990.

(27) Banci, L.; Bencini, A.; Gatteschi, D. Struct. Bond. 1982, 52, 37.

(28) Orbach, R. Proc. R. Soc. London Ser. A 1961, 264, 458.

(29) Finn, C. B. P.; Orbach, R.; Wolf, W. P. Proc. Phys. Soc. 1961, 77, 261.

(30) Thomson, S.; Luco, V.; Howe, R. Phys. Chem. Chem. Phys. 1999, 1,615 .

(31) (a) Pierloot, K.; Delabie, A.; Ribbing, C.; Verberckmoes, A. A.; Schoonheydt, R. A. J. Phys. Chem. B 1998, 102, 10789. (b) Delabie, A.; Pierloot, K.; Groothaert, M. H.; Weckhuysen, B. M.; Schoonheydt, R. A. Microporous Mesoporous Mater., in press. 